\title{
Inhibition of liver regeneration by chronic alcohol administration*
}

\author{
L DUGUAY, D COUTU, C HETU, AND J-G JOLY \\ From the Clinical Research Centre, Hôpital Saint-Luc, Department of Surgery and Medicine, Université de \\ Montréal, Montreal, Canada
}

SUMMARY Liver regeneration is the common mechanism whereby a patient recovers from a liver injury. In the western world, ethanol is the single most important aetiological factor associated with liver disease, and it appears crucial to determine if ethanol interferes with liver regeneration. We studied the response to a $70 \%$ hepatectomy in 240 rats receiving a nutritionally adequate diet containing $36 \%$ of their calories as ethanol for three weeks and their pair-fed controls receiving a liquid diet where ethanol is isocalorically replaced with carbohydrates. Criteria of liver regeneration were: incorporation of ${ }^{3} \mathrm{H}$-thymidine in hepatocyte DNA $(\mathrm{cpm} / 10 \mu \mathrm{g}$ DNA) and number of hepatocyte labelled nuclei on autoradiography per 100 high power fields. Controls displayed the usual response with peak activity of liver regeneration at 24 hours. Consumption of ethanol was associated with a statistically significant reduction of liver regeneration by both criteria for up to 72 hours after a $70 \%$ hepatectomy and delayed the peak of regenerative activity by 24 hours. This inhibiting effect was not related to the presence of alcohol in blood nor to hepatic microsomal enzyme induction by ethanol nor to widespread necrosis of hepatocytes. This effect was reversible after one week of abstinence. This impairment of liver cell renewal by ethanol may be of major significance in the severity and outcome of alcohol-related liver injury.

Liver regeneration is the common mechanism whereby a patient recovers from a liver injury, be it a trauma, an infection or a hepatotoxin. In North America the major cause of liver disease is abusive alcohol consumption which accounts for $80 \%$ of liver cirrhosis. 'In large urban areas, cirrhosis of the liver is the third major cause of deaths in patients between 35 and 54 years old. ${ }^{2}$ Currently, there is no specific therapy for cirrhosis due to alcohol excess, and treatment consists in ethanol abstinence, bed rest, vitamins, and high caloric diet; accordingly recovery from an alcohol-related liver injury depends on the capacity of the liver to regenerate. Most previous works on the mechanisms of ethanol related liver injury have mainly focused on the pathways leading to production of lesions and largely ignored the aspect of repair. It has been demonstrated that heavy alcohol consumption often leads to irreversible liver damage. . $^{3.4}$ Liver injury is explained by the combination of a number of factors deleterious to the liver: intracellular accumulation of protein and of acetaldehyde, microsomal activation of hepatotoxins, alterations in

*Supported by grants MA 6423 and PG-3 from the Medical Research Council of Canada and by a grant from the Conseil de la Recherche en Santé du Québec. Received for publication 8 July 1981 hepatic redox state, and enhancement of lymphocyte cytotoxicity. ${ }^{5}$ An additional mechanism could be through impairment of liver cell renewal; considering that this is the only known mechanism whereby a patient recovers from a liver injury, it appears crucial to determine if alcohol interferes with liver regeneration.

Previous works by other investigators have yielded conflicting results, some observing an inhibition of liver regeneration by ethanol, ${ }^{6.7}$ while others found no effect. ${ }^{8-10}$ To answer that question we have studied the response to a $70 \%$ hepatectomy in rats subjected to chronic ethanol administration.

\section{Methods}

Two hundred and forty female Sprague-Dawley rats, obtained from Canadian Breeding Farm and weighing between 130 and $150 \mathrm{~g}$, were used in all the experiments described below, Rats were fed a nutritionally adequate liquid diet containing $36 \%$ of total calories as alcohol for three weeks before surgery. Each ethanol-fed rat was pair-fed with a control partner receiving a similar diet where alcohol was isocalorically replaced with carbohydrates. ${ }^{11}$ In 
each group 20 rats were killed at 24 hour intervals for three days after surgery. Additional experimental groups were added where ethanol was removed from the diet for various periods of time before surgery, or where liver microsomal drug metabolising enzymes were induced with phenobarbitone. In these additional studies each experimental animal was pair-fed with a control rat. Details of the experimental design will be provided with the results.

\section{SURGICAL PROCEDURES}

Liver regeneration was stimulated by performing a $70 \%$ hepatectomy according to the classical technique of Higgins and Anderson. ${ }^{12}$ Operations were conducted under ether anaesthesia through a midline abdominal incision and consisted in removal of the median and left lateral lobes of the liver. This procedure constantly removes $68 \pm 2 \%$ of the liver mass. Operations were done under clean but not sterile conditions. After surgery, animals were housed in individual cages under controlled and uniform conditions of light and temperature. They had free access to their respective diet until time of killing unless otherwise stated. Animals were killed by decapitation and liver samples rapidly harvested for determination of liver regeneration.

\section{CRITERIA FOR LIVER REGENERATION}

DNA synthesis was used as the index of liver regeneration and estimated by measuring incorporation of ${ }^{3} \mathrm{H}$-thymidine into DNA and labelling of hepatocyte nuclei on autoradiographs. Two hours before killing, each animal received an intraperitoneal injection of ${ }^{3} \mathrm{H}$-thymidine (Amersham, Oakville, Ontario, $24 \mathrm{Ci} / \mathrm{nmol}$ ) in a dose of $0.2 \mathrm{mCi}$.

DNA ASSAY

DNA was extracted with hot $5 \%$ trichloroacetic acid according to Schneider and Greco, ${ }^{13}$ quantified by the diphenylamine reaction described by Burton, ${ }^{14}$ and assayed for radioactivity, after being mixed with Bray's solution, in a liquid scintillation counter (Beckham LS-8000). Results are expressed as counts per minute per 10 micrograms of DNA.

\section{LABELLED NUCLEI}

Liver specimens were fixed in $10 \%$ buffered formalin, sectioned at $5 \mu$ and stripping film autoradiographs prepared with Kodak $\mathrm{NTB}_{2}$ nuclear tract emulsion using an incubation period of 18 days, as described by McDonald and Mallory. ${ }^{15}$ Slides were then stained with haematoxylin and the number of ${ }^{3} \mathrm{H}$-thymidine labelled hepatocytes per 100 high power fields $(\times 450)$ was then counted under the microscope and used as an index of liver regeneration.
HISTOLOGY

Histological slides, $5 \mu$ thickness, were stained with haematoxylin and eosin and examined under the light microscope for evidence of inflammation and necrosis.

\section{DETERMINATION OF MICROSOMAL ENZYME} INDUCTION

Cytochrome P-450 was assayed in liver homogenates 1617 and results expressed as nmol of haemoprotein per gram of liver. ${ }^{17}$ These measurements were made in liver samples removed at the time the animals were killed.

\section{AMINOTRANSFERASES}

Serum levels of marker enzymes of liver necrosis were determined. Blood was collected at the time of surgery for determination of aspartate aminotransferase (AST) (EC 2.6.1.1) [glutamate oxalacetate transaminase (GOT)], and of alanine aminotransferase (ALT) (EC 2.6.1.2) [glutamate pyruvate transaminase (GPT)], using GOT and GPT Opt. UV-system a kits (Boehringer Mannheim, Montreal, Canada).

\section{PHENOBARBITONE}

In the group receiving phenobarbitone (phenobarbitone, BDH Pharmaceuticals, Toronto, Canada), the drug was added to the control liquid diet at a dose of $80 \mathrm{mg} / \mathrm{kg}$ per day for three weeks.

\section{ETHANOL BLOOD LEVELS}

Ethanol blood levels were determined at various intervals after alcohol abstinence in 10 pairs of rats. Measurements were made with alcohol dehydrogenase according to Bonnichsen. ${ }^{18}$

\section{Results}

To determine if chronic alcohol administration affects liver regeneration, 60 rats were fed the ethanol diet for three weeks, subjected to a $70 \%$ hepatectomy, and killed in groups of 20 at 24, 48, and 72 hours after surgery. Each experimental animal was pair-fed with a control-partner receiving the control diet and subjected to the same surgical procedure and schedule of killing. Figure 1 shows the results of the DNA uptake of ${ }^{3} \mathrm{H}$-thymidine and labelled nuclei indices in the two groups. The control animals showed the usual response to a $70 \%$ hepatectomy with peak regenerative activity occurring at 24 hours after surgery and declining thereafter. ${ }^{19}$ Chronic ethanol administration resulted in a significant and persistent reduction of liver cell renewal: peak activity was delayed to 48 hours and significantly reduced when compared with peak of controls ( $P<0.05$ for both criteria). These results suggest that chronic ethanol administration is a potent inhibitor of hepatocyte replication. 


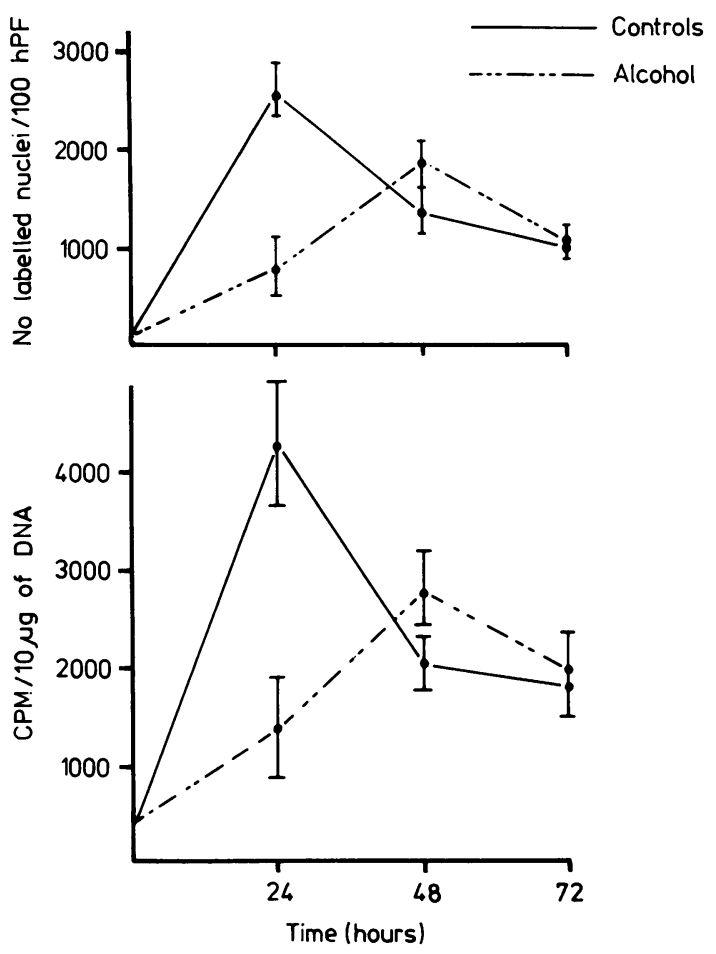

Fig. 1 Time course of liver regeneration at 24, 48, and 72 hours after a $70 \%$ hepatectomy in alcohol-treated rats (-- - ) and their pair-fed controls (-). Each point represents mean values $\pm S E M$ for 20 rats. Upper graph shows results of labelled nuclei indices, while lower graph shows results of DNA uptake of ${ }^{3} \mathrm{H}$-thymidine. Ethanol delayed the peak to 48 hours and significantly reduced the magnitude of the response $(P<0.05$ vs controls, for both criteria).

To determine if that inhibiting effect was related to the actual presence of alcohol in blood during liver regeneration, 20 rats were subjected to the same regimen as described above except that ethanol was removed from their diet 24 hours before surgery. Ethanol is rapidly metabolised by the liver and after 24 hours was no longer detectable in the blood of 10 pairs of rats in which it was determined. These results are similar to those reported by others. 20.21

These animals and their pair-fed controls were killed 24 hours after surgery. Results are shown in Fig 2. Alcohol abstinence for 24 hours before surgery did not restore the normal response to a $70 \%$ hepatectomy as measured by the usual criteria. These results suggest that the inhibiting effect observed is not dependent on the actual presence of ethanol in blood.

To determine if that inhibiting effect is permanent or reversible, 40 rats were fed the ethanol diet for three weeks, then switched to the control diet for three days (20 rats) or one week ( 20 rats), then subjected to a $70 \%$

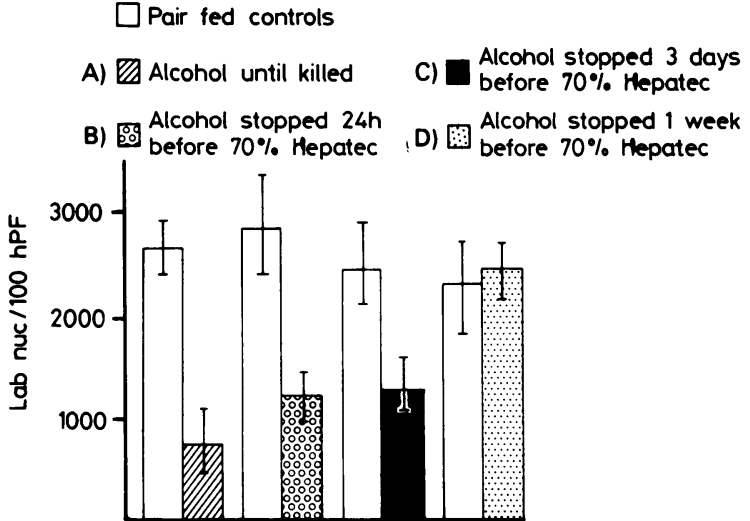

Fig. 2 Mean results of labelled nuclei indices \pm SEM in four groups of alcohol-fed rats and their pair-fed controls studied 24 hours after $70 \%$ hepatectomy. Group A.received ethanol until they were killed, and shows significant reduction of liver regeneration. Inhibition persisted although ethanol administration was stopped for 24 hours (group B) or three days (group C) before surgery. Alcohol abstinence for one week reversed the inhibiting effect to normal (group D).

hepatectomy and killed 24 hours later. In all experiments pair-fed partners were used as controls. Results are shown in Fig. 2. The inhibition of liver regeneration was persistent three days after cessation of ethanol administration $(\mathrm{P}<0.05$ vs controls $)$ but was absent after seven days of abstinence.

Ethanol is a well-known inducer of hepatocyte microsomal enzymes. ${ }^{22-24}$ This can be understood as an adaptive phenomenon, secondary to chronic alcohol consumption and useful in more efficiently removing ethanol from blood.$^{25}$ This process, like liver regeneration, involves protein synthesis. A possible explanation for the reduction of liver regeneration observed in rats fed ethanol chronically could be by competition between the two phenomena for common building blocks. To answer that question, 10 rats received the control diet to which was added phenobarbitone $(80 \mathrm{mg} / \mathrm{kg} /$ day $)$, a commonly used inducer of liver microsomal enzymes. After three weeks on phenobarbitone, they were subjected to a $70 \%$ hepatectomy and killed 24 hours later. Pair-fed partners receiving the control diet only were used as controls. As can be seen in the Table, microsomal enzyme induction was vigorous in the phenobarbitone treated animals and, as expected, reached higher levels than in the ethanol fed rats. Nevertheless, as shown in Fig. 3, rats treated with phenobarbitone displayed the normal response to a $70 \%$ hepatectomy. There was no significant difference between the experimental group and the controls, indicating that the two phenomena can take place simultaneously without one interfering with the other. 
Table Cytochrome P-450 in ethanol-fed, phenobarbitonetreated rats and their respective controls 24 hours after hepatectomy

\begin{tabular}{lcccc}
\hline & \multicolumn{4}{l}{ Alcohol and liver regeneration } \\
\cline { 2 - 5 } & Eihanol & Controls & Phenoharhitone & Controls \\
\hline $\begin{array}{l}\text { Cytochrome P-450 } \\
\text { (nmol/g liver) }\end{array}$ & & & & \\
$\begin{array}{l}\text { X SEM } \\
n\end{array}$ & $48.5 \pm 4.9$ & $34.5 \pm 2.6$ & $52.3 \pm 4.3$ & $28.2 \pm 1 \cdot 1$ \\
\hline
\end{tabular}

Microsomal enzyme induction at the time of active regeneration was significant in both experimentil groups

These results suggest that the inhibiting effect of ethanol observed in these studies is specific to alcohol and not shared with other inducers of microsomal enzymes such as phenobarbitone. Accordingly, it would appear that competition for building blocks between the two phenomena (liver regeneration and liver microsomal enzyme induction) is not the mechanism whereby ethanol inhibits liver cell renewal.

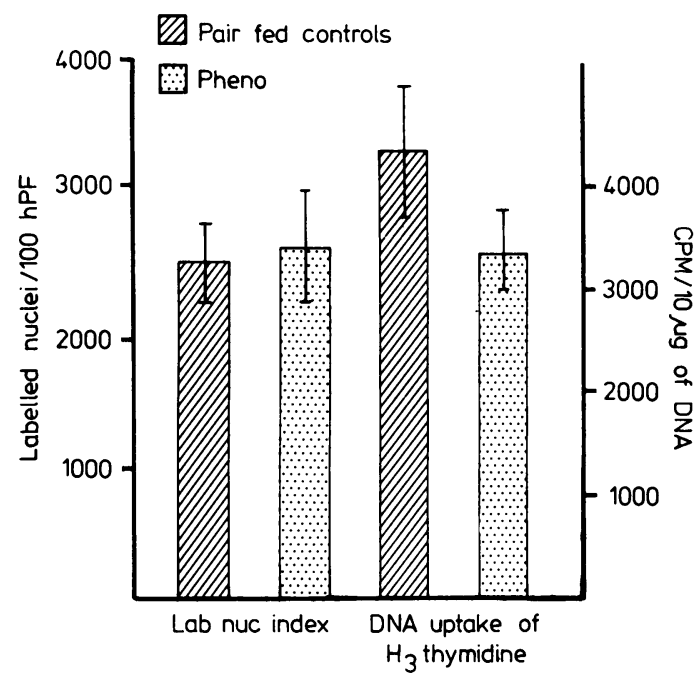

Fig. 3 Mean results $\pm S E M$ of labelled nuclei index (bars on the left) and DNA uptake of ${ }^{3} \mathrm{H}$-thymidine (bars on the right) studied 24 hours after $70 \%$ hepatectomy in 10 rats treated with phenobarbitone for three weeks and their pair-fed controls. Liver regeneration was normal in spite of vigorous microsomal enzyme induction ( $P: N S$, for both criteria).

\section{Discussion}

The results obtained in this study indicate that chronic ethanol consumption inhibits liver regeneration. The methods used to measure regeneration activity are well established and considered as the most accurate; ${ }^{26}$ the ethanol and control diets given to the animals were proposed by De Carli and Lieber in $1967^{11}$ and are now established as a standard model to study chronic alcohol consumption in the rat. It has been extensively used in our laboratory by one of us for the last eight years. Use of pair-fed controls minimises nutritional differences between the experimental and control groups.

Liver cell replacement in young adult rats after a $70 \%$ hepatectomy is a well-studied phenomenon and a reliable model extensively used to study liver regeneration; DNA synthesis requires five to eight hours to begin, 24 hours to peak, and declines progressively during the next 24 hours. ${ }^{26}$ This sequence of events is constant in healthy rats of the same strain and age subjected to similar controlled experimental conditions.

It is also apparently a maximal response in terms of timing and magnitude, as to our knowledge it cannot be significantly improved in comparable animals. While an early peak in the experimental group cannot be ruled out, it seems unlikely, as the ethanol-fed rats had no head start over the controls: alcohol by itself did not result in DNA synthesis; at time 0 the values for the ethanol and control groups were respectively $429 \pm 37$ vs $399 \pm 51$ for DNA uptake of ${ }^{3} \mathrm{H}$-thymidine and $6 \pm 0.2$ vs $7 \pm 0.2$ for labelled nuclei; the experimental group was in $\mathrm{G}_{0}$ phase at the time of hepatectomy and had to go through the entire $G_{1}$ phase in preparation for mitosis. As a large number of factors and manipulations can adversely affect the timing of the response, the study was extended to 72 hours to make sure we were not observing a simple delay in regeneration. Our data show that the peak was clearly delayed to 48 hours in the ethanol fed rat but the magnitude of the response was significantly reduced when their peak was compared with the peak of the controls.

Frank et al. ${ }^{10}$ studied the effect of acute ethanol administration upon hepatic regeneration. They observed a significant inhibition of ${ }^{3} \mathrm{H}$-thymidine incorporation into hepatic DNA of rats given ethanol by gavage every eight hours, starting immediately after a $70 \%$ hepatectomy. However, based on liver weight, protein content, and DNA content of these livers, they concluded that liver regeneration was not affected by acute alcohol administration but could offer no explanation for the discrepancy between the results of ${ }^{3} \mathrm{H}$-thymidine incorporation into DNA and those of liver cell mass determination based on weight and protein content. Liver weight, protein, and DNA content are not accurate means of measuring liver cell replacement. ${ }^{26}$ They have been estimated to be about $1 / 50$ as sensitive an index of growth as isotopic techniques and these determinations have been largely supplanted by methods that specifically measure cell divisions such as DNA uptake of ${ }^{3} \mathrm{H}$-thymidine and labelled nuclei indices. ${ }^{26}$ Besides, their conclusions, if valid, indicate that DNA replication did occur, 
without, at the same time, allowing for the incorporation of the labelled thymidine, a highly unlikely event.

Other hepatotoxins have been shown to interfere with liver cell replacement. Rosenkranz ${ }^{27}$ and associates studied liver regeneration in rats after $\mathrm{CCl}_{4}$ liver injury. They showed a significant reduction of DNA synthesis after $70 \%$ hepatectomy. In these animals mortality was high, liver necrosis widespread, but regenerative activity vigorous in the remaining hepatocytes. They concluded that the apparent diminution of liver regeneration observed in their study was an artefact related to the small number of surviving hepatocytes. Dead cells will not regenerate. The situation in our model, however, is quite different; ethanol administration as $36 \%$ of calories for three weeks was associated with minor disturbances of liver biochemistry and histology. SGOT blood levels remained normal $(41 \pm 6 \mathrm{IU} / l)$ while SGPT were slightly raised $(62 \pm 9 \mathrm{IU} / l)$. Review of our histological material showed moderate steatosis but there was no inflammation, necrosis, or fibrosis. The animals appeared to be in excellent health and operative mortality was negligible (4\%). These findings are in accordance with those of others. ${ }^{28}$ Accordingly, the impairment of liver cell renewal observed in our study cannot be explained on the basis of gross and severe hepatic necrosis. In fact, ethanol by itself appears to be a rather benign hepatotoxin; a man of average size who drinks $170 \mathrm{~g}$ ethanol per day for 25 years has only a $50 \%$ chance that cirrhosis will develop. 5.29 . In the strict, controlled, environment of the laboratory, it has been very difficult to induce cirrhosis in rats on account of ethanol consumption only; rats fed a diet providing $36 \%$ of their calories as ethanol for over a year (half their life span) will display fatty liver but will not develop irreversible liver changes. ${ }^{28}$ If ethanol by itself does not constantly produce destruction of hepatocytes, it appears to increase the vulnerability of liver cells to additional trauma, be it malnutrition, ${ }^{3031}$ infection, or chemical toxins. ${ }^{32}$ These observations have led to speculations that factors other than alcohol must be involved in alcoholic cirrhosis. $533-36$ This question is still a matter of intense interest and controversy. Activation of hepatotoxins by induced microsomal enzymes is one example of how ethanol increases the hepatotoxicity of some foreign compounds. ${ }^{3738}$ Our data suggest that inhibition of liver regeneration could be an additional mechanism whereby ethanol enhances liver vulnerability to injuries. As the capacity of a patient to recover from a liver disease depends on the capacity of his liver to regenerate, impairment of liver cell renewal by ethanol could be a factor of major significance in the severity and outcome of ethanol-related hepatic diseases. The fact that this inhibition is persistent for some time after alcohol intake has stopped and is then apparently reversible might explain the variable and unpredictable course of clinical liver failure associated with alcoholic hepatitis and cirrhosis. More studies are needed to define further if reversibility of this inhibiting effect is related to the period of exposure to alcohol and to determine the mechanism whereby ethanol impairs liver regeneration.

\section{Conclusion}

Chronic ethanol administration is associated with a significant impairment of liver regeneration. This inhibiting effect is not related to the presence of alcohol in blood nor to microsomal enzyme induction nor to widespread necrosis of hepatocytes. This effect is reversible after one week of abstinence. This impairment of liver cell renewal by ethanol may be of major significance in the severity and outcome of alcohol-related liver injury.

\section{References}

${ }^{1}$ Garceau AJ. The natural history of cirrhosis. I. Survival with oesophageal varices. $N$ Eng J Med 1963; 268:469-73.

${ }^{2}$ Vital Statistics Washington D.C.; U.S. Government Printing Office, 1974.

${ }^{3}$ Brunt PW, Kew MD, Schever PJ, et al. Studies in alcoholic liver disease in Britain. I. Clinical and pathological patterns related to natural history. Gut $1974 ; 15: 52-8$.

${ }^{4}$ Galambos JT. Natural history of alcoholic hepatitis. III. Histological changes. Gastroenterology 1972; 63:1026-35.

${ }^{5}$ Lieber CS. Pathogenesis and early diagnosis of alcoholic liver injury. N Engl J Med 1978; 298:888-93.

${ }^{6}$ Weesner R. Mendanhall C, Morgan D, et al. Suppression of liver regeneration in ethanol treated rats (Abstract). Gastroenterology 1978; 75:993.

${ }^{7}$ Wands JR, Carter EA, Bucher NLR, et al. Inhibition of liver regeneration in rats by acute and chronic ethanol intoxication. Gastroenterology 1979; 77:528-31.

${ }^{8} \mathrm{Craig}$ J. Effects of ethanol and ethionine on DNA synthesis during experimental liver regeneration. J Stud Alcohol 1973; 36:148-57.

${ }^{9}$ Chen S, Flink EB. Effects of alcohol and magnesium deficiency on hepatic regeneration (Abstract). Gastroenterology 1976; 70:956.

${ }^{10}$ Frank WO, Rayyes AN, Washington A, et al. Effect of acute ethanol administration upon hepatic regeneration. $J$ Lab Clin Med 1979; 93:402-13.

${ }^{11}$ De Carli LM, Lieber CS. Fatty liver in the rat after prolonged intake of ethanol with a nutritionally adequate new liquid diet. $J$ Nutr 1967; 91:331-6.

${ }^{12}$ Higgins GM, Anderson RM. Experimental pathology of the liver: restoration of the liver of the white rat following partial surgical removal. Arch Pathol (Chicago) 1931; 12:186.

${ }^{13}$ Schneider WC, Greco AE. Incorporation of thymidine deoxyribonucleosides into liver lipids and other components. Biochem Biophys Acta 1971; 228:610-26.

${ }^{14}$ Burton K. A study of the conditions and mechanisms of the 
diphenylamine reaction for the colorimetric estimation of deoxyribonucleic acid. Biochem J 1956; 62:315.

${ }^{15}$ McDonald RA, Mallory FK. Autoradiography using tritiated thymidine. Lab Invest 1958; 8:1547-62.

${ }^{16}$ Greim H. Naunym-Schmiedebergs Arch Pharmakol 1970; 266:261.

${ }^{17}$ Joly J-G, Doyon C, Pesant Y. Cytochrome P-450 measurement in rat liver homogenate and microsomes. Its use for correlation of microsomal losses incurred by differential centrifugation. Drug Metabol Disp 1975; 3:577-86.

${ }^{18}$ Bonnichsen R. Ethanol determination with alcohol dehydrogenase and DPN. In: Bergmeyer HU, ed. Methods of enzymatic analysis. New York: Academic Press, 1963: 285-7.

${ }^{19}$ Bucher NLR, Walt RA. Regeneration of liver and kidney. Boston: Little Brown, 1971.

${ }^{20}$ Tobon F, Mezey E. Effect of ethanol administration on hepatic and drug-metabolizing enzymes and on rates of ethanol degradation. J Lab Clin Med 1971; 77:110-21.

${ }^{21}$ Feinman L, Baraona E, Matsuzaki S, et al. Concentration dependence of ethanol metabolism in vivo in rats and man. Alcoholism: Clin Exp Res 1978; 2:381-5.

${ }^{22}$ Rubin E, Hutter F, Lieber CS. Ethanol induces hepatic smooth endoplasmic reticulum and drug-metabolizing enzymes. Science 1968; 159:1469-70.

${ }^{23}$ Rubin E, Bacchin P, Gang H, et al. Induction and inhibition of hepatic microsomal and mitochondrial enzymes by ethanol. Lab Invest 1970; 2:569-80.

${ }^{24}$ Ishii $\mathrm{H}$, Joly J-G, Lieber CS. Effect of ethanol on the amount and enzyme activities of hepatic rough and smooth microsomal membranes. Biochem Biphys Acta 1973; 291:411-20.

${ }^{25}$ Lieber CS, De Carli LM. Ethanol oxidation by hepatic microsomes: adaptive increase after ethanol feeding. Science 1968; 162:917-8.
${ }^{26}$ Bucher NLR. Regeneration of mammalian liver. Int Rev Cytol 1963; 15:245-301.

${ }^{27}$ Rosenkranz E, Charters AC, Orloff MJ. Regeneration in rat liver injured by carbon tetrachloride. Surg Forum 1975; 26:411-2.

${ }^{28}$ Lieber CS, Jones DP, Henderson J, et al. Fatty liver hyperlipemia and hyperuricemia produced by prolonged alcohol consumption and adequate dietary intake. Trans Assoc Am Physicians 1963; 76:289.

${ }^{29}$ Pequignot $\mathrm{G}$. Les enquêtes par interrogatoire permettentelles de déterminer la fréquence de l'étiologie alcoolique des cirrhoses du foie? Bull Acad Natl Med 1963; 147:90-7.

${ }^{30}$ Hall EM, Morgan WA. Progressive alcoholic cirrhosis: clinical and pathologic study of 68 cases. Arch Pathol 1939; 27:672-90.

${ }^{31}$ Lieber CS, Rubin E. Alcoholic fatty liver. $N$ Engl J Med 1969; 280:705-8.

${ }^{32}$ Joly J-G, Hétu C. Chronic ethanol administration enhances bromobenzene liver toxicity in the rat. Pharmacologist 1978; 20:158.

${ }^{33}$ Sherlock S. Causes and effects of acute liver damage. Scand J Gastroenterol 1970; 5 (suppl 6) 187-202.

${ }^{34}$ Lischner MW, Alexander JF, Galambos JI. Natural history of alcoholic hepatitis. I. The acute disease. Am J Dig Dis 1971; 16:481-94.

${ }^{35}$ Galambos JT. 'Cirrhosis'. In: Major problems in internal medicine. Philadelphia: Saunders, 1979: 3-12.

${ }^{36}$ Brunt PW. Alcohol and the liver. Gut 1971; 12:222-9.

${ }^{37}$ Hasumura Y, Teschke R, Lieber CS. Increased carbon tetrachloride hepatotoxicity and its mechanisms, after chronic ethanol consumption. Gastroenterology 1974; 66:415-22.

${ }^{38} \mathrm{Joly} \mathrm{J-G}$, Hétu C. Mechanisms of faster appearance of acetaminophen toxicity after chronic ethanol feeding. Gastroenterology 1978; 74:5 (part 2): 1158. 